\title{
Two-fold Pricing to Guarantee Individual Profits and Maximum Social Welfare in Wireless Access Networks
}

\author{
A. Hamed Mohsenian Rad, Vincent W. S. Wong, and Victor C. M. Leung \\ Department of Electrical and Computer Engineering \\ The University of British Columbia, Vancouver, Canada \\ e-mail: \{hamed, vincentw, vleung\}@ece.ubc.ca
}

\begin{abstract}
In a multi-hop wireless access network where each node is an independent self-interested commercial entity, pricing is helpful not only to encourage collaboration but also to utilize the network resources efficiently. In this paper, we propose a market-based model with two-fold pricing (TFP) for wireless access networks. In our model, the relay-pricing is used to encourage nodes to forward each other's packets. That is, each node is paid off for the relay service it provides. We also consider interferencepricing to leverage optimal resource allocation. We prove that TFP guarantees positive profit for each individual wireless node for a wide range of pricing functions. The profit increases as the node forwards more packets. Thus, the cooperative nodes are well rewarded. We then determine the relay and the interference pricing functions such that the network social welfare and the aggregate network utility are maximized. Simulation results show that, compared to a recently proposed single-fold pricing (SFP) model where only the relay prices are considered, our proposed TFP scheme significantly increases the total network profit and the network throughput. TFP also leads to more fair revenue sharing and profit distribution among the wireless relay nodes.
\end{abstract}

\section{INTRODUCTION}

Various pricing schemes have recently been proposed to encourage collaboration among the network elements or to utilize the network resources efficiently. Pricing as a tool for resource allocation was first proposed in [1] for congestion control among elastic traffic sources. In this regard, the network is designed to solve a network utility maximization (NUM) problem across all traffic sources subject to the link capacity constraints. The corresponding Lagrange multipliers are interpreted as congestion prices. Each source which uses a link resource is charged with the link's congestion price. The work in [1] has been extended to various other resource allocation problems such as medium access control, power control, and frequency channel assignment [2]-[4].

Another thread of research focuses on using pricing to encourage collaboration among the nodes [5]-[7]. In a multihop network where the nodes need to forward each other's packets, the optimal network performance might be at the cost of performance degradation for some individual intermediate relay nodes. As the intermediate nodes may have no interest in the content of the data they relay, these nodes have no reason to collaborate. This can lead to the well-known forwarder's dilemma [8], where no node forwards the packets of other nodes. To resolve this problem, incentives can be offered to the relay nodes in the form of payments or rewards in turn for their help in forwarding other nodes's traffic. In general, achieving the global optimal network performance may not be always guaranteed in the incentive-based strategies as they mainly take the individual profit objectives into consideration. In [6], He et al. proposed a pricing model for the connectivity service offered to the Internet users jointly by a group of Internet service providers (ISPs) in a fixed wired network. The pricing scheme encourages the ISPs to collaborate to achieve a fair revenue sharing. In [7], Neely proposed an economic model for stochastic wireless ad-hoc networks within the general framework of the back-pressure algorithms [9], [10], where only the relay prices are being considered. It is essentially assumed that the wireless network is interference-free. That is, the wireless nodes can transmit with an arbitrary high transmission power without interfering with each other.

In general, most of the previous pricing models have one or more of the following performance bottlenecks: (1) network resources are not efficiently allocated, (2) individual profits are not taken into consideration, and (3) interference among the wireless transmissions is not taken into account. In this paper, we address these performance bottlenecks in all three aspects. In particular, we extend the work by Neely [7] and propose a two-fold pricing (TFP) model which fully incorporates the effect of interference: relay-pricing to encourage nodes to forward each other's packets, and interference-pricing to encourage them to properly share the common network resources. We prove that for a wide range of pricing functions, our proposed TFP scheme leads to a guaranteed positive profit for each individual node. The profit increases as the node forwards more packets. This better pays off the collaborative nodes. Finally, we obtain the relay and interference pricing functions in a code division multiple access (CDMA) network such that not only the positive individual profits are guaranteed, but also the network social welfare and the aggregate network utility are maximized. Compared with the single-fold pricing (SFP) model in [7], simulation results show that our TFP scheme increases the social welfare and the network throughput by $24.6 \%$ and $14.7 \%$, respectively. It also leads to more fair revenue distributions among the collaborative wireless relay nodes as it results in $18.3 \%$ higher fairness index.

The rest of this paper is organized as follows. Our proposed market-based wireless access network model is described in Section II. The key properties of our model are analytically proved in Section III. Simulation results are presented in Section IV. Concluding remarks are given in Section V. 


\section{MARKet-BAsed Wireless Network AcCess MOdEL WITH Two-Fold PRICING}

\section{A. System Model}

Consider a stationary wireless access network and let $\mathcal{N}$, with size $|\mathcal{N}|=N$, denote the set of wireless relay nodes and $\mathcal{L}$, with size $|\mathcal{L}|=L$, denote the set of unidirectional wireless links. For each node $n \in \mathcal{N}$, the set of all incoming and outgoing links are denoted by $\mathcal{L}_{n}^{\text {in }} \subset \mathcal{L}$ and $\mathcal{L}_{n}^{\text {out }} \subset \mathcal{L}$, respectively. We also define $\mathcal{N}_{n}^{\text {in }}=\left\{m:(m, n) \in \mathcal{L}_{n}^{\text {in }}\right\}$ and $\mathcal{N}_{n}^{\text {out }}=\left\{m:(n, m) \in \mathcal{L}_{n}^{\text {out }}\right\}$ as the set of in-neighbors and the out-neighbors of node $n$, respectively. Wireless relay nodes are assumed to be independent commercial entities. Together, they form a wireless backbone to provide connectivity among wireless users in a multi-hop manner. The set of users is denoted by $\mathcal{D}$, wise size $|\mathcal{D}|=D$. Each relay node $n \in \mathcal{N}$ offers connectivity only to a subset of users, denoted by $\mathcal{D}_{n} \subset \mathcal{D}$. Each user is offered connectivity from exactly one wireless relay node. All users $i, j \in \mathcal{D}_{n}$ are able to communicate directly with each other. However, if any user $i \in \mathcal{D}_{n}$ wants to send data to another user $k \in \mathcal{D}_{c}$ where $c \in \mathcal{N} \backslash\{n\}$, it should first transfer the data to node $n$, and the data are then transferred to node $c$ via the intermediate wireless relay nodes before delivering to user $k$. In turn, node $n$ charges user $i$ for its offered connectivity service (see Section II-B). We assume that all wireless relay nodes communicate over the same frequency band which is different from those frequency bands used by the users to communicate with each other and their associated wireless relay nodes. This avoids interference between access and relay transmissions. However, the transmissions among the wireless relay nodes can still interfere with each other. A sample wireless access network is shown in Fig. 1.

Each wireless relay node $n \in \mathcal{N}$ has $N-1$ separate queues to store the incoming data according to their final destination. All data that are destined to any of the users of relay node $c \in \mathcal{N} \backslash\{n\}$ are stored in the $c^{t h}$ queue. The contents of the $c^{\text {th }}$ queue are called commodity $c$ data. For each commodity $c$ data, node $n$ maintains a set $\mathcal{H}_{n}^{(c)} \subseteq \mathcal{N}_{n}^{\text {out }}$ which includes its neighboring relay nodes with minimum hop-counts to node $c$ and can relay commodity $c$ data towards node $c$. For example, $\mathcal{H}_{e}^{(c)}=\{a, f\}, \mathcal{H}_{f}^{(c)}=\{b\}$, and $\mathcal{H}_{b}^{(c)}=\{c\}$ in Fig 1.

Time is divided into equal-length slots $\mathcal{T}=\{0,1,2, \ldots\}$. For each link $(n, m) \in \mathcal{L}$, let $\Omega_{n m}$ denote the set of all possible channel states. Channel states can vary (e.g., due to wireless fading). At each time slot $t \in \mathcal{T}$, the current channel state is denoted by $\omega_{n m}(t) \in \Omega_{n m}$. We stack up channel states of all links at time $t$ and denote the obtained $L \times 1$ vector by $\boldsymbol{\omega}(t)$. That is, $\boldsymbol{\omega}(t)=\left(w_{n m}(t), \forall n, m \in \mathcal{N},(n, m) \in \mathcal{L}\right)$. Let $\mathcal{T}_{\omega} \subseteq \mathcal{T}$ denote the time slots at which at least one of the entries of channel state vector $\boldsymbol{\omega}$ changes. We assume that $\boldsymbol{\omega}$ has an independent and identical distribution (i.i.d.) over time slots $\mathcal{T}_{\omega}$. We consider the slow fading case such that:

$$
\left|t_{2}-t_{1}\right| \geq \Lambda, \quad \forall t_{1}, t_{2} \in \mathcal{T}_{\omega}
$$

where $\Lambda \gg 1$. That is, there are at least $\Lambda$ time slots between any two consecutive changes in channel states. We will consider the fast fading case (i.e., $\Lambda \rightarrow 1$ ) in Section IV.

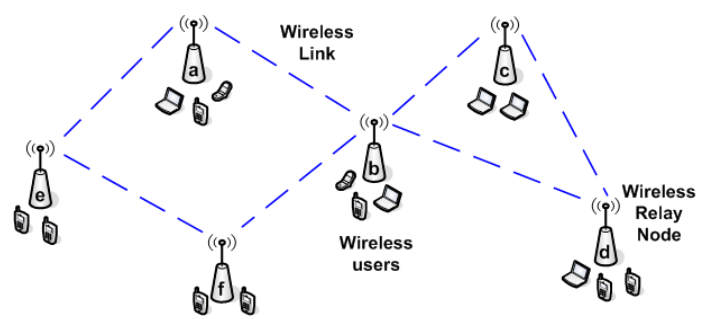

Fig. 1. A sample multi-hop wireless access network with 6 wireless relay nodes, labeled as $a, b, \ldots, f$, and 15 wireless users.

For each wireless relay node $n \in \mathcal{N}$ and any of its neighboring nodes $m \in \mathcal{N}_{n}^{\text {out }}$, let $\mu_{n m}^{(c)}(\boldsymbol{p}(t), \boldsymbol{w}(t)) \geq 0$ denote the transmission rate offered to commodity $c$ data over link $(n, m)$ during time slot $t$. Here, $\boldsymbol{p}(t)=\left(p_{n m}^{(c)}(t), \forall n, m \in\right.$ $\mathcal{N}, \forall c \in \mathcal{N} \backslash\{n\},(n, m) \in \mathcal{L})$ denotes the $L(N-1) \times 1$ vector of transmission powers for all links and all commodities. The scalar $p_{n m}^{(c)}(t) \geq 0$ denotes the transmission power corresponding to the transmission of commodity $c$ data over link $(n, m)$. In this paper, we assume that all nodes use CDMA technology. At each time slot $t \in \mathcal{T}$ and for each wireless relay node $n \in \mathcal{N}$, the commodity $c \in \mathcal{N} \backslash\{n\}$ data transmission rate over wireless link $(n, m) \in \mathcal{L}_{n}^{\text {out }}$ can be modeled as [11]:

$$
\mu_{n m}^{(c)}(\boldsymbol{p}(t), \boldsymbol{w}(t))=A_{s} \log \left(1+\frac{K h_{n m} \omega_{n m}(t) p_{n m}^{(c)}(t)}{I_{n m}\left(\boldsymbol{p}_{-n}(t)\right)+\eta_{m}}\right),
$$

where $A_{s}$ denotes the channel symbol rate, $K$ is the processing gain, $\eta_{m}$ denotes the noise power at the receiver node $m$, $h_{n m}$ is the channel gain from relay node $n$ to relay node $m, \boldsymbol{p}_{-n}(t)=\left(p_{m s}^{(d)}(t), \forall m \in \mathcal{N} \backslash\{n\}, s \in \mathcal{N}_{m}^{\text {out }}, d \in\right.$ $\mathcal{N} \backslash\{m\})$ denotes the transmission power of all nodes other than node $n$, and $I_{n m}\left(\boldsymbol{p}_{-n}(t)\right)$ denotes the aggregate interference power on link $(n, m)$. Notice that the fraction $\left(K h_{n m} \omega_{n m}(t) p_{n m}^{(c)}(t)\right) /\left(I_{n m}\left(\boldsymbol{p}_{-n}(t)\right)+\eta_{m}\right)$ is indeed the signal to interference plus noise ratio (SINR) for commodity $c$ data transmissions over link $(n, m)$. We have:

$I_{n m}\left(\boldsymbol{p}_{-n}(t)\right)=\sum_{a \in \mathcal{N} \backslash\{n\}} h_{a m}\left(\sum_{d \in \mathcal{N} \backslash\{n\}} \sum_{b \in \mathcal{N}_{a}^{\text {out }}} p_{a b}^{(d)}(t)\right)$.

Each node $n \in \mathcal{N}$ limits its total transmission power such that $\sum_{c \in \mathcal{N} \backslash\{n\}} \sum_{m \in \mathcal{N}_{n}^{\text {out }}} p_{n m}^{(c)} \leq P_{n}^{\max }$, where $P_{n}^{\max }>0$ is fixed. Thus, the transmission rates are always bounded. We define:

$$
\begin{aligned}
\mu_{n}^{\max , \text { in }} & =\max _{\boldsymbol{p}, \boldsymbol{\omega}} \sum_{c \in \mathcal{N} \backslash\{n\}} \sum_{m \in \mathcal{N}_{n}^{\text {in }}} \mu_{m n}^{(c)}(\boldsymbol{p}, \boldsymbol{w}), \\
\mu_{n}^{\max , \text { out }} & =\max _{\boldsymbol{p}, \boldsymbol{\omega}} \sum_{c \in \mathcal{N} \backslash\{n\}} \sum_{m \in \mathcal{N}_{n}^{\text {out }}} \mu_{n m}^{(c)}(\boldsymbol{p}, \boldsymbol{w}) .
\end{aligned}
$$

\section{B. Two-Fold Relay and Interference Pricing}

1) Pricing among the wireless relay nodes: In our marketbased model, at any time slot $t \in \mathcal{T}$, if node $n \in \mathcal{N}$ transmits commodity $c$ data with rate $\mu_{n m}^{(c)}(\boldsymbol{p}(t), \boldsymbol{w}(t))$ to its neighboring node $m \in \mathcal{N}_{n}^{\text {out }}$, then it pays $\mu_{n m}^{(c)}(\boldsymbol{p}(t), \boldsymbol{w}(t)) \phi_{m}^{(c)}(t)$ units of currency to node $m$ as relay service charge. Here $\phi_{m}^{(c)}(t) \geq 0$ denotes the relay price corresponding to commodity $c$ data, advertised by node $m$. In total, at time slot $t$, node $n$ pays

$$
\sum_{c \in \mathcal{N} \backslash\{n\}} \mu_{n m}^{(c)}(\boldsymbol{p}(t), \boldsymbol{w}(t)) \phi_{m}^{(c)}(t)
$$

units of currency to any neighboring relay node $m \in \mathcal{N}_{n}^{\text {out }}$ as 
relay service charge. Similarly, in total, node $n$ receives $\sum_{c \in \mathcal{N} \backslash\{n\}} \mu_{m n}^{(c)}(\boldsymbol{p}(t), \boldsymbol{w}(t)) \phi_{n}^{(c)}(t)$ units of currency from any wireless relay node $m \in \mathcal{N}_{n}^{\text {in }}$ for its offered relay service.

Besides the mutual relay services that neighboring relay nodes offer to each other, relay nodes also affect each other's operation through interference power as shown in (2) and (3). From (3), for each wireless relay node $n \in \mathcal{N}$, the higher the total transmission power $\sum_{c \in \mathcal{N} \backslash\{n\}} \sum_{m \in \mathcal{N}_{n}^{\text {out }}} p_{n m}^{(c)}(t)$, the greater is the interference power that node $n$ causes on other nodes. In our pricing model, at each time slot $t$, node $n$ pays

$$
\left(\sum_{c \in \mathcal{N} \backslash\{n\}} \sum_{m \in \mathcal{N}_{n}^{\text {out }}} p_{n m}^{(c)}(t)\right) \psi_{a}^{(n)}(t)
$$

units of currency to each node $a \in \mathcal{N} \backslash\{n\}$ as interference compensation charge. Here $\psi_{a}^{(n)}(t) \geq 0$ denotes the interference price informed by relay node $a$ to node $n$. Unlike the relay prices which vary depending on the commodity data, the interference prices are the same for all commodities as the contents of the transmissions do not affect their interference level. Instead, the interference prices may vary depending on the node locations. The closer the nodes $n$ and $m$ are located, the higher is the channel gain $h_{n a}$. This results in higher interference power and higher interference price. Similar to (7), at each time $t$, node $n$ receives $\left(\sum_{d \in \mathcal{N} \backslash\{a\}} \sum_{b \in \mathcal{N}_{a}^{\text {out }}} p_{a b}^{(d)}(t)\right) \psi_{a}^{(n)}(t)$ units of currency from node $a$ as the compensation for the interference node $a$ causes on the transmissions of node $n$.

For each relay node $n \in \mathcal{N}$ and at any time slot $t \in \mathcal{T}$, let $U_{n}^{(c)}(t)$ denote the current commodity $c \in \mathcal{N} \backslash\{n\}$ queue backlog. We define $\boldsymbol{U}(t)=\left(U_{n}^{(c)}(t), \forall n \in \mathcal{N}, \forall c \in \mathcal{N} \backslash\{n\}\right)$ as the vector of queue backlogs in all wireless relay nodes at time $t$. The relay and the interference prices are set as follows:

$$
\begin{aligned}
& \phi_{n}^{(c)}(t) \\
& =\Phi_{n}^{(c)}(\boldsymbol{U}(t-\Upsilon), \ldots, \boldsymbol{U}(t), \boldsymbol{p}(t-\Upsilon), \ldots, \boldsymbol{p}(t)), \quad \forall c \in \mathcal{N} \backslash\{n\}, \\
& \psi_{n}^{(a)}(t) \\
& =\Psi_{n}^{(a)}(\boldsymbol{U}(t-\Upsilon), \ldots, \boldsymbol{U}(t), \boldsymbol{p}(t-\Upsilon), \ldots, \boldsymbol{p}(t)), \\
& \forall a \in \mathcal{N} \backslash\{n\}
\end{aligned}
$$

where $\Phi_{n}^{(c)}(\cdot)$ and $\Psi_{n}^{(a)}(\cdot)$ are two non-negative real scalar pricing functions of all queue backlogs and all transmission powers at time slots $\{t-\Upsilon, t-\Upsilon+1, \ldots, t\}$. The above pricing functions are general and can model various relay and interference adjustment schemes. We only make a few mild assumptions. First, if $U_{n}^{(c)}(t)>0$, then $\Phi_{n}^{(c)}(\cdot)>0$. That is, if node $n$ already has some backlogged commodity $c$ data, it will not offer free relay service. Second, if $\sum_{c \in \mathcal{N} \backslash\{n\}} U_{n}^{(c)}(t)>0$ and $\sum_{c \in \mathcal{N} \backslash\{n\}} \sum_{m \in \mathcal{N}_{n}^{\text {out }}} p_{n m}^{(c)}(t)>0$, then $\Psi_{n}^{(m)}(\cdot)>0$. That is, if node $n$ has any backlog and it is currently transmitting some data on at least one of its outgoing links, it will not set its advertised interference prices to zero. Third, $\Phi_{n}^{(c)}(\cdot)$ is an increasing function of $U_{n}^{(c)}(t)$. Finally, there exists a large enough but bounded positive constant $V_{n}^{\max }$ such that for all $c \in \mathcal{N} \backslash\{n\}$ and any $t \in \mathcal{T}$, we have: $\phi_{n}^{(c)}(t) \leq V_{n}^{\max } U_{n}^{(c)}(t)$. In general, the unbounded sets of time slots at which the relay prices $\phi(t)=\left(\phi_{n}^{(c)}(t), \forall n \in \mathcal{N}, c \in \mathcal{N} \backslash\{n\}\right)$ and the interference prices $\boldsymbol{\psi}(t)=\left(\psi_{n}^{(a)}(t), \forall n \in \mathcal{N}, a \in \mathcal{N} \backslash\{n\}\right)$ are being updated are denoted by $\mathcal{T}_{\phi} \subset \mathcal{T}$ and $\mathcal{T}_{\psi} \subset \mathcal{T}$, respectively.
2) Pricing between each wireless relay node and its users: In our market-based model, each wireless relay node $n \in \mathcal{N}$ provides relay service for its associated users according to its relay prices. At each time slot $t \in \mathcal{T}$, if user $i \in \mathcal{D}_{n}$ wants to send data to user $k \in \mathcal{D}_{c}$ (for $c \neq n$ ) at rate $r_{i}^{(k)}(t)$, it needs to pay $r_{i}^{(k)}(t) \phi_{n}^{(c)}(t)$ units of currency to wireless relay node $n$ as relay service charge. At time slot $t$, in total, user $i$ pays

$$
\sum_{c \in \mathcal{N} \backslash\{n\}} \sum_{k \in \mathcal{D}_{c}} r_{i}^{(k)}(t) \phi_{n}^{(c)}(t) .
$$

We assume that relay node $n$ assigns all its users with a maximum allowed sending rate $R_{n}^{\max }$ according to its processing capacity. Each user $i \in \mathcal{D}_{n}$ also maintains a non-negative, increasing, and strictly concave utility function $g_{i}^{(k)}\left(r_{i}^{(k)}(t)\right)$ for any $k \in \mathcal{D} \backslash \mathcal{D}_{n}$ which indicates a monetary measure of user $i$ 's level of satisfaction from sending rate $r_{i}^{(k)}(t)$. Thus, user $i$ adjusts its rates $\boldsymbol{r}_{i}=\left(r_{i}^{(k)}(t), \forall k \in \mathcal{D} \backslash \mathcal{D}_{n}\right)$ by solving the following local optimization problem:

$$
\begin{aligned}
\max _{\boldsymbol{r}_{i}(t) \succeq \mathbf{0}} & \sum_{c \in \mathcal{N} \backslash\{n\}} \sum_{k \in \mathcal{D}_{c}}\left(g_{i}^{(k)}\left(r_{i}^{(k)}(t)\right)-r_{i}^{(k)}(t) \phi_{n}^{(c)}(t)\right) \\
\text { s.t. } & \sum_{c \in \mathcal{N} \backslash\{n\}} \sum_{k \in \mathcal{D}_{c}} r_{i}^{(k)}(t) \leq R_{n}^{\max } .
\end{aligned}
$$

Notice that the objective function of the above optimization problem is always non-negative as at least for $\boldsymbol{r}_{i}=\mathbf{0}$ it is equal to zero. We define user $i$ 's profit at each time slot $t \in \mathcal{T}$ as:

$$
\begin{aligned}
\vartheta_{i}(t)= & \left(\sum_{n \in \mathcal{N} \backslash\{n\}} \sum_{k \in \mathcal{D}_{c}} g_{i}^{(k)}\left(r_{i}^{(k)}(t)\right)\right) \\
& -\left(\sum_{n \in \mathcal{N} \backslash\{n\}}\left(\sum_{k \in \mathcal{D}_{c}} r_{i}^{(k)}(t)\right) \phi_{n}^{(c)}(t)\right) .
\end{aligned}
$$

From (9), user $i$ adjusts $\boldsymbol{r}_{i}(t)$ to maximize its profit subject to the total rate constraint imposed by relay node $n$. Unlike the network model in [7] where each relay node can only support at most one user, here we allow each wireless relay node to offer the connectivity service to an arbitrary number of users.

\section{Resource Allocation and Routing}

At each time slot $t \in \mathcal{T}$, given the advertised relay prices from all its out-neighbors, node $n \in \mathcal{N}$ can compute differential relay price for any $m \in \mathcal{N}_{n}^{\text {out }}$ and each $c \in \mathcal{N} \backslash\{n\}$ as [7]:

$$
\delta_{n m}^{(c)}(t)=\phi_{n}^{(c)}(t)-\phi_{m}^{(c)}(t)-\phi^{\max },
$$

where $\phi^{\max }=V^{\max } U^{\max }$ denotes the largest possible change in any relay price during one time slot. Here, $V^{\max }=$ $\max _{n} V_{n}^{\max }$ and $U^{\max }=\max _{n}\left\{\mu_{n}^{\max \text {, out }}, \mu_{n}^{\max \text {, in }}+R_{n}^{\max }\right\}$ represents the largest possible change in any queue backlog where $\mu_{n}^{\max \text {, in }}$ and $\mu_{n}^{\max \text {, out }}$ are defined in (4) and (5), respectively. At the beginning of each time slot $t \in \mathcal{T}$, relay node $n$ measures $\omega_{n m}(t)$ for all its outgoing wireless links $(n, m) \in$ $\mathcal{L}_{n}^{\text {out }}$ and adjusts $\boldsymbol{p}_{n}(t)=\left(p_{n m}^{(c)}(t), \forall c \in \mathcal{N} \backslash\{n\}, m \in \mathcal{N}_{n}^{\text {out }}\right)$ by solving the following local optimization problem:

$$
\begin{aligned}
\max _{\boldsymbol{p}_{n}(t) \succeq \mathbf{0}} & \sum_{c \in \mathcal{N} \backslash\{n\}} \sum_{m \in \mathcal{N}_{n}^{\text {out }}} \mu_{n m}^{(c)}(\boldsymbol{p}(t), \boldsymbol{\omega}(t)) \delta_{n m}^{(c)}(t)- \\
& \left(\sum_{c \in \mathcal{N} \backslash\{n\}} \sum_{m \in \mathcal{N}_{n}^{\text {out }}} p_{n m}^{(c)}(t)\right)\left(\sum_{a \in \mathcal{N} \backslash\{n\}} \psi_{a}^{(n)}(t)\right) \\
\text { s.t. } \quad & \sum_{c \in \mathcal{N} \backslash\{n\}} \sum_{m \in \mathcal{N}_{n}^{\text {out }}} p_{n m}^{(c)}(t) \leq P_{n}^{\max }, \\
& p_{n m}^{(c)}(t)=0, \quad \forall c \in \mathcal{N} \backslash\{n\}, m \notin \mathcal{H}_{n}^{(c)} \\
& \text { or } c \neq c_{n m}^{\star}(t) \text { or } \delta_{n m}^{(c)}(t) \leq 0,
\end{aligned}
$$


where $\mu_{n m}^{(c)}$ is as in (2), $\mathcal{H}_{n}^{(c)}$ is defined in Section II-A, and

$$
c_{n m}^{\star}(t)=\arg \max _{c: m \in \mathcal{H}_{n}^{(c)}} \delta_{n m}^{(c)}(t), \quad \forall n \in \mathcal{N}, m \in \mathcal{N}_{n}^{\text {out }} .
$$

The optimal objective function in (12) is always non-negative as at least for $\boldsymbol{p}_{n}(t)=\mathbf{0}$ it is equal to 0 . Comparing to the resource allocation problem in [7], the objective function in (12) has an extra negative term $-\left(\sum_{c \in \mathcal{N} \backslash\{n\}} \sum_{m \in \mathcal{N}_{n}^{\text {out }}} p_{n m}^{(c)}(t)\right)$ $\left(\sum_{a \in \mathcal{N} \backslash\{n\}} \psi_{a}^{(n)}(t)\right)$, which denotes the total interference compensation charge that node $n$ should pay to other relay nodes. By solving (12), node $n$ finds the trade-off between maximizing $\sum_{c \in \mathcal{N} \backslash\{n\}} \sum_{m \in \mathcal{N} \text { out }} \mu_{n m}^{(c)}(\boldsymbol{p}(t), \boldsymbol{\omega}(t)) \delta_{n m}(t)$ (i.e., the original objective function in [7]) and minimizing its interference compensation cost. Each node then implements the same routing strategy as in [7]. That is, node $n$ transmits commodity $c_{n m}^{\star}(t)$ data on link $(n, m)$ if $\delta_{n m}^{\left(c_{n m}^{\star}(t)\right)}>0$. No commodity $c \neq c_{n m}^{\star}(t)$ data is sent on link $(n, m)$ at time $t$.

Theorem 1: Let $\boldsymbol{p}_{n}^{\star}(t)$ denote the optimal solution of problem (12). Assuming that $K \gg 1$ and all links operate in the high SINR regime (cf. [3], [11]), for each $m \in \mathcal{N}_{n}^{\text {out }}$ and any $c \in \mathcal{N} \backslash\{n\}$, if $c=c_{n m}^{\star}(t), \delta_{n m}^{(c)}(t)>0$, and $m \in \mathcal{H}_{n}^{(c)}$, then:

$$
p_{n m}^{\star(c)}(t)=\min \left\{\frac{\delta_{n m}^{\left(c_{n m}^{\star}(t)\right)}(t)}{\sum_{a \in \mathcal{N} \backslash\{n\}} \psi_{a}^{(n)}(t)}, \frac{\delta_{n m}^{\left(c_{n m}^{\star}(t)\right)}(t) P_{n}^{\max }}{\sum_{a \in \mathcal{N}_{n}^{\text {out }}} \delta_{n a}^{\left(c_{n a}^{\star}(t)\right)}}\right\} ;
$$

otherwise, $p_{n m}^{\star(c)}(t)=0$.

Theorem 1 provides a closed-form solution for problem (12). The proof of Theorem 1 is omitted for brevity. Knowing that problem (12) is transformable to a convex problem using the logarithmic change of variables (cf. [3, Theorem 1]), it is easy to verify that (14) satisfies the necessary and sufficient Karush-Kuhn-Tucker (KKT) optimality conditions (cf. [12]).

\section{Guaranteed Positive Individual Relay Profits AND MAXIMUM SOCIAL WELFARE}

Recall that each relay node $n \in \mathcal{N}$ is a commercial entity. Thus, it is interested in making money out of its offered relay services. At each time slot $t \in \mathcal{T}$ we define node $n$ 's profit as:

$$
\begin{aligned}
\chi_{n}(t)= & \sum_{c \in \mathcal{N} \backslash\{n\}} \sum_{i \in \mathcal{D}_{n}} \sum_{k \in \mathcal{D}_{c}} r_{i}^{(k)}(t) \phi_{n}^{(c)}(t) \\
& +\sum_{m \in \mathcal{N}_{n}^{\text {in }}} \sum_{c \in \mathcal{N} \backslash\{n\}} \mu_{m n}^{(c)}(\boldsymbol{p}(t), \boldsymbol{w}(t)) \phi_{n}^{(c)}(t) \\
& -\sum_{m \in \mathcal{N}_{n}^{\text {out }}} \sum_{c \in \mathcal{N} \backslash\{n\}} \mu_{n m}^{(c)}(\boldsymbol{p}(t), \boldsymbol{w}(t)) \phi_{m}^{(c)}(t) \\
& +\sum_{a \in \mathcal{N} \backslash\{n\}} \psi_{n}^{(a)}(t) \sum_{d \in \mathcal{N} \backslash\{a\}} \sum_{b \in \mathcal{N}_{a}^{\text {out }}} p_{a b}^{(d)}(t) \\
& -\sum_{a \in \mathcal{N} \backslash\{n\}} \psi_{a}^{(n)}(t) \sum_{c \in \mathcal{N} \backslash\{n\}} \sum_{m \in \mathcal{N}_{n}^{\text {out }}} p_{n m}^{(c)}(t) .
\end{aligned}
$$

The first term in (15) is the total relay charges from all users $i \in \mathcal{D}_{n}$. The second and the third terms denote the total relay charges from and to all the neighboring nodes, respectively. The fourth and the fifth terms denote the total interference charges from and to all other nodes $a \in \mathcal{N} \backslash\{n\}$, respectively. We are now ready to present our first key result:

Theorem 2: For each $T \gg 1$ and any relay node $n \in \mathcal{N}$,

$$
\begin{aligned}
& \sum_{t=0}^{T} \chi_{n}(t) \\
& \geq \sum_{t=0}^{T} \sum_{a \in \mathcal{N} \backslash\{n\}} \psi_{n}^{(a)}(t)\left(\sum_{d \in \mathcal{N} \backslash\{a\}} \sum_{b \in \mathcal{N}_{a}^{\text {out }}} p_{a b}^{(d)}(t)\right) .
\end{aligned}
$$

The proof of Theorem 2 is given in Appendix A. From Theorem 2, each node is guaranteed to obtain an individual profit at least as high as the right-hand side (RHS) of (16). It is clear that all the terms in the RHS of (16) are non-negative. From the assumptions in Section II-B, the RHS of (16) can be zero only if for the duration from time slot $t=0$ to time slot $t=T$, no relay node in set $\mathcal{N} \backslash\{n\}$ transmits any data and there is no data in any of the $N-1$ queues in relay node $n$. This can happen only if either $N=1$ and there does not exist any other relay node in the network or node $n$ has set its relay prices too high so that none of its users and neighboring relay nodes are interested in transferring their data to node $n$. The former is indeed the case where there is no need to relay node $n$ as all users in set $\mathcal{D}_{n}=\mathcal{D}$ can communicate with each other directly. The latter also implies the case where node $n$ is reluctant to contribute as a part of the wireless access network.

Corollary 1: Each wireless relay node that contributes in relaying data is guaranteed to receive a positive-valued profit.

Theorem 2 and Corollary 1 are general and apply to any choice of user utilities and pricing functions. Next, we determine the pricing functions $\Phi_{n}^{(c)}$ and $\Psi_{n}^{(m)}$ for all relay nodes $n \in \mathcal{N}$, any commodity $c \in \mathcal{N} \backslash\{n\}$, and any neighboring node $m \in \mathcal{N}_{n}^{\text {out }}$ to maximize the network social welfare; i.e., the aggregate profit across all wireless relay nodes and users:

$$
\sum_{t=1}^{T} \sum_{n \in \mathcal{N}} \chi_{n}(t)+\sum_{t=1}^{T} \sum_{i \in \mathcal{D}} \vartheta_{i}(t) .
$$

Lemma 1: Replacing $\vartheta_{i}(t)$ and $\chi_{n}(t)$ in (17) by (10) and (15), respectively, the social welfare model in (17) reduces to:

$$
\sum_{t=1}^{T} \sum_{n \in \mathcal{N}} \sum_{i \in \mathcal{D}_{n}} \sum_{c \in \mathcal{N} \backslash\{n\}} \sum_{k \in \mathcal{D}_{c}} g_{i}^{(k)}\left(r_{i}^{(k)}(t)\right) .
$$

That is, the monetary exchanges among the wireless relay nodes and the wireless users cancel out each other.

The summation in (18) is indeed the aggregate network utility across all users. Thus, from Lemma 1, maximizing the network social welfare in our TFP model is equivalent to maximizing the aggregate network utility. This helps us to present our second key result as follows.

Theorem 3: Given $\mathcal{T}$ (i.e., the set of time slots), $\mathcal{T}_{\omega}$ (i.e., the set of time slots at which the channel states change), and $\Lambda \gg 1$ (i.e., the fading parameter), let $\mathcal{T}_{\phi}=\mathcal{T}_{\omega}, \mathcal{T}_{\psi}=\mathcal{T}$, and $\Upsilon=\Lambda$ where $\mathcal{T}_{\omega}, \mathcal{T}_{\psi}$, and $\Upsilon$ are defined in Section II-B. The aggregate network utility and the network social welfare are maximized if each node $n \in \mathcal{N}$ at each time slot $t^{\prime} \in \mathcal{T}_{\phi}$ set:

$$
\Phi_{n}^{(c)}=V U_{n}^{(c)}\left(t^{\prime}\right), \quad \forall c \in \mathcal{N} \backslash\{n\},
$$

and then at each time slot $t \in\left\{t^{\prime}, \ldots, t^{\prime}+\Upsilon\right\} \subset \mathcal{T}_{\psi}$ set:

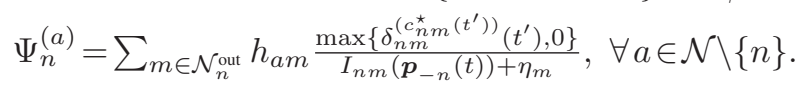

Here $V>0$ is an arbitrary design parameter.

The sketch of the proof of Theorem 3 is given in Appendix B. Theorems 2 and 3 together show that if the transmission powers, relay prices, and the interference prices are set according to (14), (19), and (20), respectively, then not only each relay node receives a guaranteed positive profit, but also the network social welfare and the network utility are maximized. 


\section{Simulation Results}

We evaluate the performance of our proposed TFP scheme and compare it with a recently proposed SFP scheme in [7] where only the relay prices are taken into account and the network is assumed to be interference-free. We consider three performance metrics: 1) network social welfare, 2) fairness index, and 3) aggregate network throughput. The fairness index is calculated among the profits that the wireless relay nodes achieve. It is obtained as [13]:

$$
\frac{\left(\sum_{n \in \mathcal{N}} \sum_{t=1}^{T} \chi_{n}(t)\right)^{2}}{N \sum_{n \in \mathcal{N}}\left(\sum_{t=1}^{T} \chi_{n}(t)\right)^{2}},
$$

where $T=5000$ is the simulation time and profit $\chi_{n}(t)$ is as in (15). Each relay node $n$ provides the connectivity for $\left|\mathcal{D}_{n}\right|=5$ users and each user is interested in sending data to two other users inside the network. We consider 10 different random topologies. In each topology, the wireless relay nodes are randomly located in a $1 \mathrm{~km} \times 1 \mathrm{~km}$ square field and the communication range is $200 \mathrm{~m}$. For each wireless relay node $n \in \mathcal{N}$, we have: $P_{n}^{\max }=20 \mathrm{~W}$ and $R_{n}^{\max }=100 \mathrm{kbps}$. The transmission power, relay prices, and the interference prices are set as in (14), (19), and (20), respectively. The unit of currency is selected such that for a unit queue backlog, relaying $1 \mathrm{Mbps}$ data costs 1 cent (i.e., 0.01 dollar).

The social welfare where the fading parameter $\Lambda=10$ and the number of wireless relay nodes $N$ varies from 10 to 50 are shown in Fig. 2. Each point is the average of the measurements for all 10 simulated topologies. We can see that our proposed TFP is always superior compared to the SFP scheme in [7]. The performance gain increases as $N$ increases. It is because our interference pricing scheme better leverages optimal resource allocation. As the number of nodes increases, more nodes interfere with each other's transmissions and optimal interference control becomes more crucial. In fact, as Theorem 3 shows, our TFP model leads to maximum social welfare. Considering the case where $N=50$, TFP results in $24.6 \%$ higher network social welfare compared to SFP.

The exact value of the social welfare, fairness index, and aggregate throughput for each of the 10 simulated topologies when $N=50$ are shown in Fig. 3. We consider both slow fading (SF) channels where the fading parameter $\Lambda=10$ as well as the fast fading (FF) channels where $\Lambda=2$. From Fig. 3 (a), TFP always results in higher social welfare compared to SFP. However, in the presence of FF channels, TFP cannot achieve optimal performance. Recall that Theorem 3 requires $\Lambda$ to be large and it only works for SF channels. On the other hand, from Fig. 3 (b), TFP always acts more fair compared to SFT, regardless of the fading parameter $\Lambda$. Recall that Theorem 2 is indeed independent of any system parameter. It guarantees high positive profit for all nodes. In fact, having the interference prices helps those wireless relay nodes which do not experience high traffic demand. Instead, they make some money out of the interference charges. On average, TFP results in $18.3 \%$ and $35.2 \%$ higher fairness index compared to SFP in the presence of SF and FF channels, respectively. From

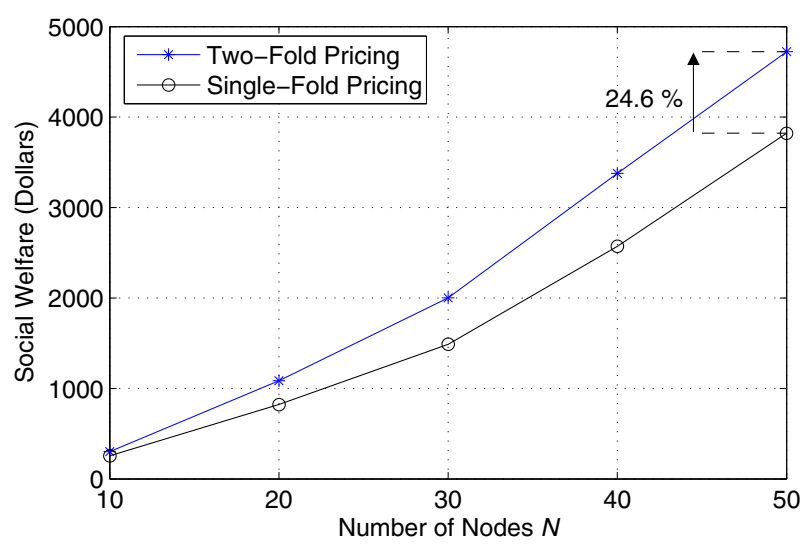

Fig. 2. Network social welfare when the number of wireless relay nodes $N$ varies from 10 to 50 . Each wireless relay node supports 5 wireless users.
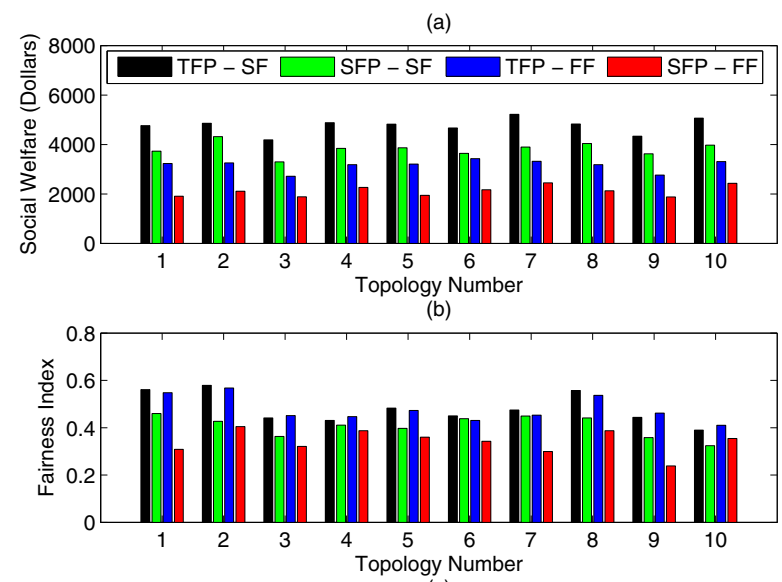

(c)

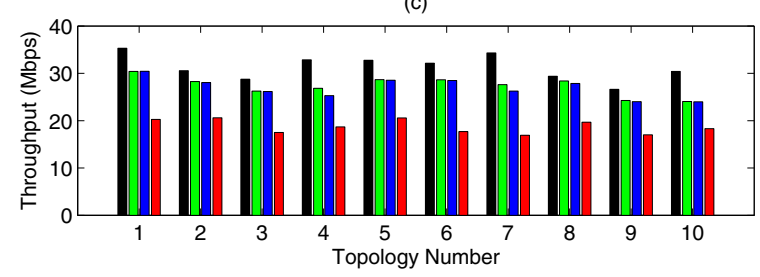

Fig. 3. Simulation results for each of the 10 random topologies when $N=50$ : (a) Social welfare, (b) Fairness Index, and (c) Aggregate Throughput.

Fig. 3 (c), TFP also always results in higher throughput. On average, the aggregate network throughput increases by $14.7 \%$ and $32.4 \%$ compared to SFP in the presence of SF and FF channels, respectively. In summary, our proposed TFP scheme leads to not only higher aggregate profit across the nodes and users, but also more fair revenue distribution among the relay nodes. It also results in a higher network throughput.

\section{CONCLUSION}

In this paper, we proposed a market-based wireless access network model with two-fold pricing (TFP) where several self-interested wireless relay nodes provide the connectivity for various utility-holding wireless users. The relay-prices are used as incentives to encourage nodes to collaborate and forward each other's packets. The interference-prices are also 
used to leverage optimal resource allocation. Together, the relay and interference prices incorporate both cooperative and competitive interactions among the nodes. The positive and increasing profit for each individual relay node is guaranteed for a wide range of pricing functions. Assuming that CDMA technology is being used for transmissions, the relay and the interference pricing functions are then determined to not only guarantee individual profits but also maximize the aggregate network throughput and the network social welfare. Compared with the SFP scheme in [7], simulation results show that TFP increases the network social welfare and the aggregate network throughput by $24.6 \%$ and $14.7 \%$, respectively. It also leads to more fair revenue sharing among the wireless relay nodes as it results in $18.3 \%$ higher fairness index. For future work, we shall consider the effect of user mobility and roaming.

\section{APPENDIX}

\section{A. Proof of Theorem 2}

For the ease of exposition, for any link $(n, m) \in \mathcal{L}$ and each commodity $c \in \mathcal{N} \backslash\{n\}$ we replace $\mu_{n m}^{(c)}(\boldsymbol{p}(t), \boldsymbol{\omega}(t))$ by $\mu_{n m}^{(c)}(t)$. From (11) and (15), for any node $n \in \mathcal{N}$ and at any time $t \in \mathcal{T}$, $\chi_{n}(t)=\chi_{n}(t)+\sum_{c \in \mathcal{N} \backslash\{n\}} \sum_{m \in \mathcal{N}_{n}^{\text {out }}} \mu_{n m}^{(c)}(t) \delta_{n m}^{(c)}(t)$

$-\sum_{c \in \mathcal{N} \backslash\{n\}} \sum_{m \in \mathcal{N}_{n}^{\text {out }}} \mu_{n m}^{(c)}(t)\left(\phi_{n}^{(c)}(t)-\phi_{m}^{(c)}(t)-\phi^{\max }\right)$ $=\left[\sum_{c \in \mathcal{N} \backslash\{n\}} \sum_{m \in \mathcal{N}_{n}^{\text {out }}} \mu_{n m}^{(c)}(t) \delta_{n m}^{(c)}(t)\right.$

$$
\left.-\sum_{a \in \mathcal{N} \backslash\{n\}} \psi_{a}^{(n)}(t) \sum_{c \in \mathcal{N} \backslash\{n\}} \sum_{m \in \mathcal{N}_{n}^{\text {out }}} p_{n m}^{(c)}(t)\right]
$$$$
+\left[\sum_{c \in \mathcal{N} \backslash\{n\}}\left(\sum_{i \in \mathcal{D}_{n}} \sum_{k \in \mathcal{D}_{c}} r_{i}^{(k)}(t)+\sum_{m \in \mathcal{N}_{n}^{\text {in }}} \mu_{m n}^{(c)}(t)\right) \phi_{n}^{(c)}(t)\right.
$$$$
\left.-\sum_{c \in \mathcal{N} \backslash\{n\}} \sum_{m \in \mathcal{N}_{n}^{\text {out }}} \mu_{n m}^{(c)}(t)\left(\phi_{n}^{(c)}(t)-\phi^{\max }\right)\right]
$$

Since the optimal objective function in (12) is non-negative,

$$
\begin{aligned}
& \sum_{c \in \mathcal{N} \backslash\{n\}} \sum_{m \in \mathcal{N}_{n}^{\text {out }}} \mu_{n m}^{(c)}(\boldsymbol{p}(t), \boldsymbol{\omega}(t)) \delta_{n m}^{(c)}(t) \\
& -\sum_{a \in \mathcal{N} \backslash\{n\}} \psi_{a}^{(n)}(t) \sum_{c \in \mathcal{N} \backslash\{n\}} \sum_{m \in \mathcal{N}_{n}^{\text {out }}} p_{n m}^{(c)}(t) \geq 0 .
\end{aligned}
$$

Following the proof of [7, Theorem 2b], we can show that:

$$
\begin{aligned}
& \sum_{c \in \mathcal{N} \backslash\{n\}}\left(\sum_{i \in \mathcal{D}_{n}} \sum_{k \in \mathcal{D}_{c}^{r}} r_{i}^{(k)}(t)+\sum_{m \in \mathcal{N}_{n}^{\text {in }}} \mu_{m n}^{(c)}(t)\right) \phi_{n}^{(c)}(t) \\
& -\sum_{c \in \mathcal{N} \backslash\{n\}} \sum_{m \in \mathcal{N}_{n}^{\text {out }}} \mu_{n m}^{(c)}(t)\left(\phi_{n}^{(c)}(t)-\phi^{\max }\right) \geq 0 .
\end{aligned}
$$

By replacing (23) and (24) in (21) we have:

$$
\chi_{n}(t) \geq \sum_{a \in \mathcal{N} \backslash\{n\}} \psi_{n}^{(m)}(t) \sum_{d \in \mathcal{N} \backslash\{a\}} \sum_{b \in \mathcal{N}_{a}^{\text {out }}} p_{a b}^{(d)}(t) .
$$

Adding up both sides for $t=1, \ldots, T$, Eq. (16) is resulted.

\section{B. Sketch of the Proof of Theorem 3}

Given $t^{\prime} \in \mathcal{T}_{\phi}$, we can check the cross-derivatives of the update equations in (14) and (20) during time slots $\left\{t^{\prime}, \ldots, t^{\prime}+\Upsilon\right\}$ and show that these update equation have increasing differences and together form a super-modular game (cf. [14], [15]). Thus, $\boldsymbol{p}(t)$ and $\boldsymbol{\psi}(t)$ converge to some fixed point $\left\langle\boldsymbol{p}^{\star}, \boldsymbol{\psi}^{\star}\right\rangle[15$, Theorem 1a]. By definition, $\boldsymbol{p}^{\star}$ should denote the optimal solution of the local problem in (12) for all nodes. We can verify that $\boldsymbol{p}^{\star}$ also satisfies the KKT conditions corresponding to the following global optimization problem:

$$
\begin{array}{clr}
\max _{p(t) \succeq \mathbf{0}} & \sum_{n \in \mathcal{N}} \sum_{c \in \mathcal{N} \backslash\{n\}} \sum_{m \in \mathcal{N}_{n}^{\text {out }}} \mu_{n m}^{(c)}(\boldsymbol{p}(t), \boldsymbol{\omega}(t)) \delta_{n m}^{(c)}(t) \\
\text { s.t. } & \sum_{c \in \mathcal{N} \backslash\{n\}} \sum_{m \in \mathcal{N}_{n}^{\text {out }}} p_{n m}^{(c)}(t) \leq P_{n}^{\max }, \quad \forall n \in \mathcal{N} \\
& p_{n m}^{(c)}(t)=0, \quad \forall n \in \mathcal{N}, c \in \mathcal{N} \backslash\{n\}, m \notin \mathcal{H}_{n}^{(c)} \\
& \text { or } c \neq c_{n m}^{\star}(t) \text { or } \delta_{n m}^{(c)}(t) \leq 0 .
\end{array}
$$

Notice that the objective function in (26) is different from the objective function in the local problem (12) as it is the weighted summation of all link rates. Using the logarithmic change of variables (cf. [3]), we can transform problem (26) to an equivalent convex problem. Hence problem (26) has a unique KKT point $\boldsymbol{p}^{\star}$ which is indeed its optimal solution (cf. [12]). Thus, assuming that $\Upsilon=\Lambda$ is large enough, the update equations in (14) and (20) converge to the optimal solution of problem (26). This, together with (19), results in achieving maximum network utility (cf. [10] and [16, Theorem 4]). From Lemma 1, it also implies maximum social welfare.

\section{ACKNOWLEDGMENT}

This work was supported by Bell Canada and the Natural Sciences and Engineering Research Council of Canada.

\section{REFERENCES}

[1] F. P. Kelly, "Charging and rate control for elastic traffic," European Trans. on Telecommunication, vol. 8, pp. 33-37, 1997.

[2] L. Chen, S. Low, and J. Doyle, "Joint congestion control and media access control design for ad hoc wireless networks," in Proc. of IEEE INFOCOM, Miami, Florida, March 2005.

[3] M. Chiang, "To layer or not to layer: Balancing transport and physical layers in wireless multihop networks," in Proc. of IEEE INFOCOM, Hong Kong, China, Mar. 2004.

[4] A. H. Mohsenian Rad and V. W. S. Wong, "Joint channel allocation, interface assignment, and mac design for multi-channel wireless mesh networks," in Proc. of IEEE INFOCOM, Anchorage, Alaska, May 2007.

[5] O. Ileri, S. Mau, and N. Mandayam, "Pricing for enabling forwarding in self-configuring ad-hoc networks," IEEE J. on Selected Areas in Communications, vol. 23, pp. 151-162, Jan. 2005.

[6] L. He and J. Walrand, "Pricing and revenue sharing strategies for internet service providers," IEEE J. on Selected Areas in Communications, vol. 24, pp. 942-951, May 2006.

[7] M. J. Neely, "Optimal pricing in a free market wireless network," in Proc. of IEEE INFOCOM, Anchorage, Alaska, May 2007.

[8] M. Felegyhazi and J. P. Hubaux, "Game theory in wireless networks: A tutorial,” EPFL Technical Report, LCA-REPORT-2006-002, Feb. 2006.

[9] L. Tassiulas and A. Ephremides, "Stability properties of constrained queueing systems and scheduling policies for maximum throughput in multihop radio networks," IEEE Trans. on Automatic Control, vol. 13, pp. 582-595, June 1992.

[10] M. J. Neely, E. Modiano, and C. P. Li, "Fairness and opimal stochastic control for heterogeneous networks," in Proc. of IEEE INFOCOM, Miami, Florida, Mar. 2005.

[11] D. Tse and P. Viswanath, Fundamentals of Wireless Communication. Cambridge University Press, 2004.

[12] S. Boyd and L. Vandenberghe, Convex Optimization. Cambridge University Press, 2004.

[13] R. Jain, W. Hawe, and D. Chiu, "A quantitative measure of fairness and discrimination for resource allocation in shared computer systems," Tech. Rep. DEC-TR-301, Sept. 1984.

[14] D. M. Topkis, Supermodularity and Complementarity. Princeton University Press, 1998.

[15] J. Huang, R. A. Berry, and M. L. Honig, "Distributed interference compensation for wireless networks," IEEE J. on Selected Areas in Communications, vol. 24, pp. 1074-1084, May 2006.

[16] Y. Xi and E. Yeh, "Throughput optimal distributed control of stochastic wireless networks," in Proc. of WiOpt, Boston, MA, Apr. 2006. 